\title{
Evolution of pathogen response genes associated with increased disease susceptibility during adaptation to an extreme drought in a Brassica rapa plant population
}

Niamh Breda O'Hara ( $\square$ niamh.ohara@cornell.edu )

Jacobs Technion-Cornell Institute, Cornell Tech

Steven J Franks

Fordham University

Nolan C Kane

University of Colorado Boulder

Silas Tittes

University of Colorado Boulder

Joshua S Rest

Stony Brook University

\section{Research article}

Keywords: Climate change, Brassica rapa, Alternaria brassicae, drought, resurrection approach, necrotrophic fungal pathogen response.

Posted Date: August 8th, 2020

DOI: https://doi.org/10.21203/rs.2.16267/v2

License: (c) (i) This work is licensed under a Creative Commons Attribution 4.0 International License.

Read Full License

Version of Record: A version of this preprint was published at BMC Ecology and Evolution on April 21st, 2021. See the published version at https://doi.org/10.1186/s12862-021-01789-7. 


\section{Abstract}

BACKGROUND Pathogens are key components in natural and agricultural plant systems. There is evidence of evolutionary changes in disease susceptibility as a consequence of climate change, but we know little about the underlying genetic basis of this evolution. To address this, we took advantage of a historical seed collection of a Brassica rapa population, which we previously demonstrated evolved an increase in disease susceptibility to a necrotrophic fungal pathogen following a drought.

RESULTS Previously, we combined a resurrection experiment with genome-wide sequencing of 124 pooled ancestral and descendant plants. Here, using these previously generated sequence data (Franks et al, 2016), we show that well-characterized necrotrophic fungal pathogen response (NFPR) genes have evolved, as indicated by changes in allele frequency, between ancestors and descendants, with several of them identified as extreme $F_{S T}$ outliers. The jasmonic acid (JA) signaling pathway in particular seems to underlie the evolution of disease susceptibility, in addition to its well characterized role in plastic disease response. We identify a list of 260 genes that are both NFPR genes and are differentially expressed in response to drought, based on publicly available data. We present evidence that five of these genes evolved between ancestors and descendants, suggesting that the drought acted as the evolutionary driver, and that the accompanying increase in disease susceptibility may have been a consequence of genetic pleiotropy.

CONCLUSIONS Our study provides evidence that for this population, standing variation in NFPR genes is affected by natural selection related to climate change. Our results reveal potentially important candidates that may underlie trait evolution in both crops and natural systems. Additionally, this trade-off between adaptation to biotic and abiotic stresses is an example of how climate change can have diverse and unexpected consequences.

\section{Background}

Pathogens exert strong selection on plants, often causing them to evolve increased resistance. Simultaneously, plant systems are exposed to a myriad of other selective pressures. Persistent climate change, such as long-term drought, may result in evolution of the hosts, pathogens, or both, leading to changes in virulence or susceptibility (Desprez-Loustau et al., 2006; Garrett et al., 2006; Pautasso et al., 2012; Garrett et al., 2014; O'Hara et al., 2016b). More broadly, exploration of how multiple, and at times opposing, selective pressures shape the genomes of plants is an important area of ongoing research.

The genetics of plant pathogen response is exceedingly complex and specific to whether the pathogen is necrotrophic or biotrophic (Glazebrook, 2005; Mengiste, 2012). While response to biotrophs has been extensively studied and found to be generally mediated by a salicylic acid-dependent pathway, response to necrotrophic pathogens (the focus here) is less well understood and tends to have a more complex genetic architecture. Necrotrophic pathogens, which first kill tissue and then extract nutrients (Agrios, 2005), stimulate a response pathway generally mediated by jasmonic acid (JA) and ethylene (ET) 
(McDowell and Dangl, 2000). Such biotic stress response pathways have been shown to interact with abiotic stress response pathways. In particular, abscisic acid (ABA), which regulates growth and development in response to water availability, has been shown to suppress biotic stress response pathways, leaving plants more susceptible to pathogens (Mauch-Mani and Mauch, 2005). ABA is thus an example of a regulatory system that may potentially be involved in adaptation to drought (Shinozaki et al., 2003), and which may also result in pleiotropic evolution of pathogen susceptibility.

The most common approach used to characterize the genes responsible for pathogen response are expression studies in model plant-pathogen systems. However, low genetic variation, and distant phylogenetic relationships often limit the translation of such studies to crop breeding or characterization of evolving natural systems. Therefore, there is great promise in studying plant-pathogen genetics of natural systems. Some progress has been made in plant-pathogen systems with a simple genetic architecture including exploring genetic variation in resistance in natural populations for the LinumMelampsora system (Thrall and Burdon, 2003) and variation in specific resistance genes such as the RPW8 (Recognition of Powdery Mildew)(Jorgensen and Emerson, 2008). But thus far, variation in resistance has been mapped at a spatial scale looking at local adaptation, rather than being followed over time (Thrall and Burdon, 2003; Laine et al., 2011; Pautasso et al., 2012). The lack of focus on evolutionary trajectories limits our ability to predict evolutionary responses over short time scales. We addressed this limitation by taking advantage of evolutionary events, which allowed us to compare the genomes of ancestors and descendants to identify the genes involved in evolutionary changes in disease susceptibility in a natural system.

The system we used is the well-studied example of the adaptive evolution of earlier flowering in the annual herbaceous plant, Brassica rapa L. (syn. campestris)(Brassicaceae, field mustard) in response to a natural, extended drought (Franks et al., 2007; Franks and Weis, 2008; Franks, 2011). Previous work used a resurrection approach (Franks et al., 2008; Franks et al., 2018) in which B. rapa seed was collected in southern California in 1997, before an extended drought, and then again in 2004, post-drought. Preand post-drought seeds were then grown under common conditions in the greenhouse and challenged with Alternaria brassicae. A. brassicae, which causes Alternaria blackspot disease, is a necrotrophic pathogenic sac fungus that causes damping off, leaf spots, defoliation and reduced seed yield (Koike et al., 2006). We previously found that this population experienced a genetically-based evolutionary increase in Alternaria blackspot disease susceptibility (O'Hara et al., 2016b), together with an adaptive shift to avoid drought through earlier flowering (Franks et al., 2007). While the shift to earlier flowering and drought avoidance was adaptive, the drivers for an increase in disease susceptibility remain unclear. Pathogen susceptibility may have evolved either due to direct selection, or indirectly as a result of selection on common genes (Penuelas and Filella, 2001; Hoffmann and Sgro, 2011; Franks et al., 2014; O'Hara et al., 2016b).

To explore the shift in disease susceptibility following a drought, we hypothesized that: 1) known necrotrophic fungal pathogen response genes (including on JA and ET pathways) evolved, and 2) pleiotropic genes, underlying pathogen and drought response evolved. To test these hypotheses, we used 
genome sequences of an ancestral (pre-drought) and descendant (post-drought) B. rapa natural population, and explored whether necrotrophic fungal pathogen response (NFPR) genes evolved. We assessed signatures of evolution in two complementary ways. First, we conducted genome-wide outlier $\mathrm{F}_{\mathrm{ST}}$ analysis to determine if allele frequencies of previously characterized NFPR genes were differentiated between ancestors and descendants, indicating rapid contemporary evolution at these loci. Second, we used site frequency spectrum analysis (Tajima's D) to look for historical signatures of selective sweeps or other strong or recurrent selective patterns at NFPR genes. Finally, we identified a list of genes that were both NFPR genes and were differentially expressed in response to drought, based on publicly available data. We used this list to consider the potential role of pleiotropic evolution of both NFPR and drought response.

\section{Methods}

\section{PLANT SAMPLES}

Previously, a large number of seeds $(>10,000)$ were bulk collected from a natural feral $B$. rapa population (Arboretum) in Southern California near the University of California Irvine campus. Seeds were collected along a transect from ripened seedpods (siliques) in 1997, before an extended natural drought, and then again in 2004, post-drought. Plants were grown from seed and cross-pollinated in the greenhouse within year to control for maternal effects (Franks et al., 2007). Shortly before this study, F1 seeds were grown in the greenhouse and cross-pollinated within year to create F2 plants and control for storage effects.

\section{SAMPLE PREPARATION AND SHOTGUN SEQUENCING}

Using the methods described in more detail in Franks et al. (2016), previously, 500 ancestral and descendant plants (total $N=1000$ ) were grown up from $F 2$ seeds on light carts. Tissue was collected from first true leaves, dried using silica beads, and lysed (Fastprep, MP Bio). Genomic DNA was extracted (DNeasy Plant Mini Kit, Qiagen), checked for quality and quantified by real-time PCR using GAPDH as a reference gene (Untergasser et al., 2007; Qi et al., 2010). Samples with high molecular weight were pooled in equimolar amounts within year (ancestral $N=50$, descendant $N=74$ ). Duplicate pools were created to verify that pooling was precise, for a total of 4 pools. Pools were used to make Nextera (Illumina) libraries using the manufacturer's protocol. Each pool was tagged and sequenced in 1/3 lane of a HiSeq 2000 (Illumina). Note that Franks et al. (2016) also included a second population (Back Bay) not studied here, and for which we do not have disease susceptibility data. We have focused on the Arboretum population for disease susceptibility studies (O'Hara et al., 2016b) due to the greater evolutionary shifts in multiple traits experienced by that population (Franks et al., 2007).

BIOINFORMATIC ANALYSIS AND VALIDATION

We previously used computational methods described in Franks et al. (2016) to trim, clean and prepare our reads for analysis. Briefly, we trimmed ends of reads with a quality score of less than 20, filtered low complexity regions, and removed library barcodes. BWA (Li and Durbin, 2010) was used to align 
sequence reads to the $B$. rapa genome version 1.18 (http://brassicadb.org/brad/)(Wang, 2011). Sequence variants were called using the Bayesian mpileup algorithm in Samtools (Li et al., 2009; Li and Durbin, 2010). Population genetic estimates were calculated using the packages Popoolation (Pandey et al., 2011) and Popoolation2 (Kofler et al., 2011), including divergence $\left(F_{S T}\right)$ (Hartl and Clark, 2007; Karlsson et al., 2007) and Tajima's D (Tajima, 1989). Population genetic estimates were compared between pooling replicates and found to be highly correlated, so these technical replicates were merged for all reported analyses.

In previous work, to validate SNPs and population genetic estimates determined using these pooled whole-genome sequences, we selected 10 genes that were found to be highly differentiated between ancestors and descendants and assayed a single SNP within each gene for 116 individuals (29 individuals from each population and time point) using Kompetitive Allele-Specific PCR genotyping (KASP; LGC Genomics). We found a strong correlation between allele frequencies obtained from KASP genotyping compared to pooled Illumina sequencing (Pearson correlation $r=0.83, p<0.001$ )(Franks et al., 2016).

Statistical significance was determined for the $\mathrm{F}_{\mathrm{ST}}$ values of genes by comparing the experimental $\mathrm{F}_{\mathrm{ST}}$ distribution to a null distribution created by resampling 1000x with bootstrapping. The experimental distribution was regressed on the null distribution and $p$ values were calculated for residuals using the car package in R (Fox and Weisberg, 2011; R Core Team, 2013). Multiple correction was conducted using fdrtool (Strimmer, 2008) and genes were considered significant if they had a q value of less than 0.05 (Storey and Tibshirani, 2003). A q value cutoff of 0.05 implies that about $5 \%$ of the genes deemed significant are expected to be false positives. We also report the top 50 most differentiated NFPR genes, including those with a q value of $>0.05$.

To calculate Tajima's D, we used the Variance-sliding.pl script from Poopolation with some changes to the default parameters including setting a more stringent minimum coverage of $8 \mathrm{x}$.

\section{PLANT-PATHOGEN SYSTEM}

We used the compatible plant-pathogen system Brassica rapa - Alternara brassicae in this study, as described in detail in our previous work (O'Hara et al., 2016b). The molecular mechanisms of pathogen response in the Brassica rapa - Alternaria brassicae system is not well understood (Lawrence et al., 2008), however, extensive research has been conducted on the closely related plant pathogen system Arabidopsis thaliana-Alternariabrassicicola, which is a model system used for studying diseases caused by necrotrophs (van Wees et al., 2003; Glazebrook, 2005; Mukherjee et al., 2009). In the current study, we took advantage of this extensive model system work and used an a priori approach (Marra et al., 2012) to characterize the evolution of a set of well-researched candidate disease-related genes in our resurrected ancestral and descendent natural populations, as described in more detail in the next section. Studies in the $A$. thaliana-A. brassicicola system have identified a large number $(\sim 1,100)$ of candidate NFPR genes (van Wees et al., 2003; Glazebrook, 2005; Mukherjee et al., 2009). Also, these studies found that JA is 
required for resistance, indicated by susceptibility in mutants with non-functional copies of genes involved in the JA pathway, including a mutation in the downstream CO/1 gene (see Glazebrook 2005 for further details). Additionally, a recent $B$. rapa expression study was used to identify candidate drought response genes (Guo et al., 2017).

\section{FUNCTIONAL ANALYSIS}

In the current study, for our a priori analysis approach, a database of 1,182 well-characterized candidate NFPR genes (Additional file 2) was assembled from two previous $A$. thaliana-A. brassicicola studies by Mukherjee et al. (2009) and van Wees et al. (2003); results from these studies were used for functional annotations in our study. These studies were chosen in order to span the range of compatibility that exists between B. rapa-A. brassicae (Nowicki et al., 2012), including genes shown to be involved in both compatible interactions (Mukherjee et al., 2009) and incompatible interactions (van Wees et al., 2003). Briefly, to identify candidate NFPR genes, van Wees et al. (2003) used mutants with non-functional copies of genes involved in JA and SA signaling followed by inoculation and expression profiling, and Mukherjee et al. (2009) used suppression subtractive hybridization (SSH) to identify differential expression between compatible and incompatible plant lines challenged with the pathogen. For both studies, which measured differential expression across the whole transcriptome, NFPR candidate genes were determined as genes with differential expression between plants that were inoculated with the fungal pathogen and those that received a mock inoculation. We used this database for functional annotations in our study.

We next considered 7,076 genes candidate drought response genes (Additional file 3) which were differentially expressed in Guo et al. (2017) study in response to a polyethylene glycol (PEG) treatment, as compared to controls (no PEG), and that had a $>2$ fold change at 12 hours post-treatment. We then identified genes differentially expressed in common among both this drought study and the NFPR studies (above). In total, we found 260 genes were significantly differentially expressed in both types of conditions.

Lastly, we conducted a functional analysis by analyzing gene ontology (GO) using ErmineJ (Gillis et al., 2010), with annotations from Arabidopsis thaliana. To analyze our $\mathrm{F}_{\mathrm{ST}}$ distribution, which was nonnormal, we used ranked gene scores in a receiver operator characteristic (ROC) analysis and default ErmineJ settings. $P$ values were corrected using a Bejamini-Hochberg false discover rate (FDR) correction.

\section{Results}

Previously we identified 5,812,602 SNPs (out of 235,128,010 sites), including SNPs in 35,202 genes using a low-coverage approach ( $25 x$ per pooled library). The general results of our sequencing and our observation of global and flowering time related allele frequency shifts have been published elsewhere (Franks et al., 2016). For this study, we analyzed candidate NFPR genes in order to explore the genomics of an observed evolutionary shift in disease susceptibility for this population following the drought event 
(O'Hara et al., 2016b). We found evidence of evolution in NFPR genes, demonstrated by a change in allele frequencies at these pathogen response loci between ancestor and descendant populations, and that the JA signaling pathway seemed to underlie the evolution of disease susceptibility. We also found that a number of these NFPR genes were also responsive to drought, suggesting the potential role of pleiotropic evolution.

\section{EVOLUTION OF NFPR AND NFPR/DROUGHT RESPONSE GENES}

To explore our first hypothesis, that pathogen response genes evolved, we used an a priori approach, and found that 11 genes from of our database of 1,182 candidate NFPR genes were significantly differentiated between ancestors and descendants (Figure 1), with an $\mathrm{F}_{\mathrm{ST}}$ above background compared to all the genes, as determined by outlier $F_{S T}$ analysis $(q<0.05)$. These genes were found throughout the genome on the majority of $B$. rapa's 10 chromosomes, including chromosomes $1,2,4,5,6,7$, and 9 . In contrast, in the entire genome, we saw 434 out of 35,202 genes evolved (Franks et al., 2016). The NFPR genes that evolved were not over-represented among all genes that showed significant evolution (Chisquare $=0.846, p=0.358$ ). The mean $F_{S T}$ for all NFPR genes was not significantly elevated above background (ANOVA: $F_{1,35199}=0.008, P=0.929$ ). In a Manhattan plot comparing $F_{S T}$ of NFPR to all other genes across the genome, the NFPR and all gene's trend lines tracked one another (Figure 1), in fact 95\% confidence intervals (not shown) overlapped across the whole genome. We report the 20 most differentiated NFPR genes (Table 1; see Additional file 1 for top 50). This includes the 11 genes that were significant, plus additional genes that could potentially have been involved in the shift in susceptibility despite not being statistically significant after false discovery correction.

To explore our second hypothesis, that genes involved in both NFPR and drought response evolved, we also used an a priori approach and found that $5(2.0 \%)$ genes from our joint list of 260 genes that responded to both conditions were significantly differentiated between ancestors and descendants, with an $F_{S T}$ above background compared to all genes $(q<0.05)$ (Table 1). These five genes did not constitute an over-representation among all genes that showed significant evolution (Chi-square $=0.974, p=0.324$ ). We show the overlap in NFPR, drought response, and genes that evolved (demonstrated by significant $F_{S T}$ values) in a Venn diagram (Figure 3). This list of genes that evolved, and that are associated with both NFPR and drought response, are candidates genes for pleiotropic evolution (i.e. where genetic change at a single locus affected both phenotypes).

\section{HISTORICAL SELECTION}

To determine if the genes that were highly differentiated between 1997 and 2004 were also under selection historically, we calculated the Tajima's $D$ value for each gene. We found that none of the highly differentiated genes also had a significantly reduced Tajima's $D$ value. There was no difference in 
Tajima's $D$ between significantly differentiated $\left(F_{S T}\right)$ NFPR genes and the rest of the NFPR genes (ANOVA: ancestors $F_{1,1180}=2.398, P=0.122$; descendants $F_{1,1180}=0.551, P=0.458$ ). This was consistent with our genome-wide result, where we found little overlap between genes with high $\mathrm{F}_{\mathrm{ST}}$ and negative Tajima's D (Franks et al., 2016). These findings were not surprising because these estimates detect selection acting at different time scales. Visual inspection of Tajima's D values over the genome suggested that three of our significant $\left(F_{S T}\right)$ NFPR genes were in regions of reduced Tajima's D (red bars in Figure 1), which could indicate they were linked to regions that may have experienced historical selective sweeps, although the values of Tajima D for the genes of interest were themselves not significantly reduced.

We next explored the nature of segregating sites in two NFPR candidate genes with the highest $\mathrm{F}_{\mathrm{ST}}$ values, Bra030295 and Bra038107. We explored whether polymorphism in these genes would result in changes in the amino acid sequence, and therefore potentially be a direct subject of selection. We made alignments of the ancestral, descendant, and reference sequences for these genes (Figure 2). We determined whether sites were in exons or introns and, if in exons, whether they were synonymous or nonsynonymous. These genes were also in regions that had reduced Tajima's $D$, by visual inspection, or have important functions (see Discussion). We found that the two genes together had 10 SNPs in exons, and 5 of these exonic SNPs were nonsynonymous. Bra030295 had two and Bra038107 had three nonsynonymous sites that experienced an allele shift between ancestors to descendants.

\section{JASMONIC ACID AND THE EVOLUTION OF DISEASE SUSCEPTIBILITY}

Since the JA pathway is such an important mediator of necrotrophic fungal pathogen response, we specifically explored whether JA (and downstream gene CO/1) may be involved in the evolution of disease susceptibility. We found that 17 (34\%) out of our top 50 most NFPR differentiated genes were COI1 and/or JA dependent (Additional file 1). This is similar to the ratio 441 (37\%) out of 1,182 of COI1/JA genes which are differentially expressed in response to pathogens in van Wees et al. (2003) and Mukherjee et al. (2009) pathogen studies; we found that these ratios are statistically equivalent (Chisquare $=0.225, p=0.635$ ), despite the very different approach of analysis using resurrection/sequencing instead of gene expression.

\section{GENE ONTOLOGY ANALYSIS}

We used gene ontology analysis to determine if pathogen response functional categories are overrepresented globally among all genes when ranked by their level of evolutionary differentiation between ancestors and descendants $\left(\mathrm{F}_{\mathrm{ST}}\right)$. We found that pathogen response categories were not significantly enriched and were not highly ranked out of the 3,136 categories. The top pathogen response category was "plant-type hypersensitive response" (G0:0009626), ranked 54th ( $P=0.014$, Benjamini- 
Hochberg FDR corrected $P=0.835)$, followed closely by "host programmed cell death induced by a symbiont" (GO:0034050), ranked 56th $(P=0.015$, Benjamini-Hochberg FDR corrected $P=0.822)$.

\section{Discussion}

In previous work, we found that a natural B. rapa plant population in Southern California evolved earlier flowering (Franks et al., 2007) and increased fungal disease susceptibility (O'Hara et al., 2016b) following a 7-year natural drought. A shift in susceptibility for this plant pathogen system could have widespread implications because this fungus is an important agricultural problem for $B$. rapa crops (Rotem, 1994; Meena et al., 2010). Also, we have found widespread infection (22.2\% of plants observed to be infected) in all 6 of our B. rapa field populations on the West Coast (O'Hara et al., 2016a). In the current study, we compared the genomics of ancestral and descendant plants from one population to elucidate the underlying genetic and molecular basis of this shift in disease susceptibility. This is the first study, to our knowledge, to examine the genetic basis of evolution of susceptibility to pathogens in a natural plant population following drought (Mitchell-Olds and Schmitt, 2006; Metcalf and Mitchell-Olds, 2009; Walsh, 2009; Rosas et al., 2014). There are many challenges to studying evolution in natural systems. Using a resurrection genomics approach we were able to both directly measure the disease susceptibility of ancestors and descendants in common conditions, and directly assess evolution by measuring changes in allele frequencies between these ancestral and descendant populations (Franks et al., 2016).

It should be noted that this was an observational study focused on one natural population that underwent an evolutionary shift in response to an extended drought. Experimental evolution studies with additional populations, including a non-drought control population, would be a powerful approach to address some of these findings further.

\section{EVOLUTION OF NFPR GENES}

Using an outlier $\mathrm{F}_{\mathrm{ST}}$ analysis approach, we found evidence for our first hypothesis, that a number of NFPR genes evolved over the course of just 7 years of drought. This is consistent with selection acting on a trait that is controlled by multiple loci, such as defense response to a necrotrophic fungus. This is expected to be more complex than response to a biotrophic pathogen, which in many cases is controlled by a single gene (Glazebrook, 2005; Nowicki et al., 2012). We did not see significant evolution across the wider NFPR system compared to the rest of the genome. It is possible, given the genetically complex nature of NFPR, that specific causative genes could evolve, underlying a shift in susceptibility, without evolution across the wider system, especially given the short time frame between ancestors and descendants studied. In addition, we found that a substantial proportion of these NFPR genes are JA/COI1 dependent. Studies in the closely related $A$. thaliana- . brassicicola model plant pathogen system have identified COI1dependent genes, part of the JA signalling pathway, as playing a central role in defense against necrotrophic fungal pathogens, suggesting that JA signalling mediates the defense response. JA activity is dependent on the downstream COI1 gene, which encodes an F-box protein involved in proteolysis (Xie 
et al., 1998). JA signalling might play an important role in the evolution of susceptibility in addition to its known role in a plastic response to necrotrophic fungal pathogens.

There are limitations to using an outlier $\mathrm{F}_{\mathrm{ST}}$ analysis to characterize the ultimate molecular or developmental mechanisms by which a population phenotypically evolves (e.g. driving mechanism of evolution). However, our outlier $\mathrm{F}_{\mathrm{ST}}$ approach, together with a q-value threshold correction, is effective at characterizing loci that showed significant shifts, and thereby can identify putative candidate genes underlying genetically-based phenotypic shifts observed. This approach has also been successfully utilized in other studies in these populations (Franks et al., 2016).

\section{POTENTIAL SIGNATURES OF PLEIOTROPIC EVOLUTION}

In previous work, we found adaptive evolution in response to drought (through earlier flowering) also resulted in the evolution of pathogen susceptibility in this population. In exploration of this associated response, in the current study, we also found evidence for our second hypothesis: that a number of genes involved in both drought and pathogen response evolved, suggesting a signature of pleiotropic evolution. In the current study, we considered genes to be candidates for pleiotropic evolution for drought and necrotrophic fungal pathogens if they had been found to be differentially expressed in response to independent drought stress and inoculation with a necrotrophic fungal pathogen, and if in our study they evolved between ancestors and descendants. A subset of five of these genes evolved in our study. Allelic variation in any of these five genes may have simultaneously affected both drought response and NFPR response, i.e. caused pleiotropic phenotypic changes. For this population we found clear evidence that JA pathway genes, which affect both pathogen response, flowering time, and stress resistance (Zhou et al., 2005; Wu et al., 2008; Garg et al., 2012), evolved, suggesting evidence for evolution in pleiotropic genes in this system. These findings agree with other studies which have found that pleiotropy plays a role in shaping multiple ecological traits for plant populations displaying adaptation to local water availability (McKay et al., 2003). Furthermore, it is possible that antagonistic pleiotropy was playing a role in the evolution observed (Williams, 1957). Under this scenario, pleiotropic genes underlying both drought and pathogen response could have evolved under positive selection due to drought, despite negative selection by pathogens, if the negative selection was not strong enough to overcome the positive selection, or if it was not acting in the contemporary environment (O'Hara et al., 2016b). Indeed, the increased susceptibility we observed in this population was accompanied by an evolutionary shift to thinner leaves (increased specific leave area)(O'Hara et al., 2016b), which could indicate a trade-off between drought escape and disease susceptibility in agreement with the growth and defense trade-off (Simms and Rausher, 1987; Mole, 1994).

This study was limited due to it being an observational study of a single population. Alternative explanations to pleiotropy include that drought response and pathogen susceptibility were acted on separately, or that if there is a large cost of disease resistance and these pathogens become less common during a drought, then selection could favor reduced resistance due to these costs. The current study design does not allow us to differentiate between these alternative explanations. Finally, in addition 
to pleiotropy, correlated change in these two phenotypes could have been caused by drift, linkage, or by simultaneous change in selective pressure on both flowering time and pathogen load. While we cannot eliminate these from consideration, they likely played a minor role in the observed patterns due to data presented in a previous study showing an adaptive evolution in response to drought, lack of evidence of a bottleneck, and the short 7-year time frame over which the evolution occurred, too short for drift alone to explain the extensive allelic differentiation observed (Franks et al., 2016).

\section{HISTORICAL SELECTION}

In addition to examining evolution following the recent drought, we tested whether NFPR genes were under historical directional selection by looking for genes with a low Tajima's $D(<-2)$. Loci under balancing selection would not have a low Tajima's D. Genome-wide, we found no association between $F_{S T}$ and Tajima's $D$, and there were no genes that were outliers for both high $F_{S T}(>0.2)$ and low Tajima's $D$ (<-2) (Franks et al., 2016). This indicates that evolution in response to the drought affected different genes in this population than have historically been the target of directional selection.

\section{TOP CANDIDATE GENES UNDERLYING EVOLUTIONARY SHIFT IN SUSCEPTIBILITY}

The most highly differentiated gene in this study was Sec14p-like phosphatidylinositol transfer family protein (Bra030295) (Figure 2A; $F_{S T}=0.21 ; q=1.75 \times 10^{-5}$ ) which has been found to be differentially expressed in Arabidopsis in response to infection with A. brassicicola (van Wees et al., 2003) and cabbage leaf curl virus (Ascencio-lbanez et al., 2008). Little is known about the specific function of this gene, although it is thought to play a role in the movement of substances across cell membranes and has been found to be expressed widely in 23 plant structures (Lamesch et al., 2011). The shift in allele frequency at two non-synonomous segregating sites in this gene indicate a potential functional effect.

Several of the NFPR genes with the highest level of differentiation between ancestors and descendants demonstrate the pleiotropic nature of the genes subject to evolution in response to the drought in this population. We found that the second most highly differentiated NFPR gene was the COI1 dependent gene ERF4 (Bra038107) $\left(F_{S T}=0.20 ; q=7.30 \times 10^{-5}\right)$. In addition to a demonstrated role in the antagonistic regulation of JA and ET, this gene is also induced by ABA (Hoth et al., 2002; Nemhauser et al., 2006). This is interesting because this gene, along with other genes mediated by ABA that we found to have high $\mathrm{F}_{\mathrm{ST}}$, including Bra012746 and Bra033968, and Bra011282, have pleiotropic roles in both abiotic and biotic stress response (Journot-Catalino et al., 2006; Huang et al., 2008; Liu et al., 2011). Studies have shown that ERF4 is induced by ABA, and ERF4 suppresses expression of PDF1.2 (McGrath et al., 2005), a defense effector that is elicited by JA signaling (Glazebrook, 2005). This gene showed a shift in allele frequency at three non-synonomous sites, and evolution at these sites could have a functional effect (Figure 2B).

Caffeoyl-CoA 3-O-methyltransferase (Bra033968) is an NFPR gene that is CO/1 dependent (van Wees et al., 2003) and also had a significant $F_{S T}$ value. It is a lignin biosynthesis gene, which is pleiotropic, 
playing a role in both drought and pathogen response. Lignification is a primary defense mechanism against pathogens (Vance et al., 1980), which works primarily through leaf structure (Taiz and Zeiger, 2006). It has also been shown to be suppressed in Arabidopsis by ABA application, along with other pathogen response genes, resulting in a decrease in lignin accumulation and an increase in disease susceptibility to Pseudomonas syringae pathovar (pv.) tomato (Mohr and Cahill, 2007). This gene is also interesting because lignin plays a key role in leaf structure, and we found evidence that leaf structure played a role in the increase in disease susceptibility seen (O'Hara et al., 2016b), with earlier flowering plants having thinner leaves that displayed greater disease severity.

\section{Conclusions}

The results from our resurrection genomics study demonstrate that many well-characterized candidate NFPR genes evolved, with a change in allele frequency, in just 7 years of drought. We conclude that COI and the JA pathway are likely to be involved in the evolution of disease susceptibility in this population. These genes are thus candidates for future investigation of variation in disease susceptibility in other crop and wild populations of this and related species. Several implicated genes are potentially involved in both defense and drought resistance, suggesting that antagonistic pleiotropy potentially caused the observed evolutionary increase in disease susceptibility following a natural drought. That this previously unexplained increase in disease susceptibility is a result of adaptation to drought represents a surprising response of a plant population facing changes in local climate.

\section{Declarations}

\section{ETHICS APPROVAL AND CONSENT TO PARTICIPATE}

All fungal work referenced in O'Hara et al 2016 was conducted in sterile conditions, and was permitted under APHIS license \#P526P-11-00130.

\section{CONSENT FOR PUBLICATION}

Not applicable.

\section{COMPETING INTERESTS}

The authors have no conflict of interest to declare. 


\section{AVAILABILITY OF DATA AND MATERIAL}

The dataset supporting the conclusions of this article are included within the article (and its additional files).

\section{FUNDING}

Funding was provided by a fellowship from AAUW and the Elisha M. Friedman Postdoctoral Fellowship to N. O'Hara, a grant (DEB-1142784) from the National Science Foundation to S. Franks, and a grant (R01GM108904) from NIGMS-NIH to J.S.R.

\section{AUTHOR CONTRIBUTIONS}

All authors designed the experiments; NO managed the project, analysis, and conducted the laboratory work; NO lead the writing of the manuscript and all authors contributed to writing and editing; all authors contributed to data analyses; and all authors have read, edited, and approved of the final manuscript.

\section{ACKNOWLEDGEMENTS}

We thank A.E. Weis and E. Austen for providing seeds, R. Welt for growing plants and DNA extraction, and G. Amidi-Abraham for laboratory assistance.

\section{References}

Agrios, G.N. (2005). Plant Pathology. Burlington, MA: Academic Press.

Ascencio-lbanez, J., Sozzani, R., Lee, T., Chu, T., Wolfinger, R., Cella, R., et al. (2008). Global analysis of Arabidopsis gene expression uncovers a complex array of changes impacting pathogen response and cell cycle during geminivirus infection. Plant Physiology 148, 436 - 454.

Desprez-Loustau, M.L., Marcais, B., Nageleisen, L., Piou, D., and Vannini, A. (2006). Interactive effects of drought and pathogens in forest trees. Annals of Forest Science 63, 597-612. 
Fox, J., and Weisberg, S. (2011). "An R Companion to Applied Regression". 2 ed. (Thousand Oaks, CA: Sage).

Franks, S., Hamann, E., and Weis, A.E. (2018). Using the resurrection approach to understand contemporary evolution in changing environments. Evolutionary Applications 11(1), 17-28.

Franks, S., Kane, N., O'Hara, N., Tittes, S., and Rest, J. (2016). Rapid genome-wide evolution in Brassica rapa populations following drought revealed by sequencing of ancestral and descendant gene pools. Molecular Ecology 25(15), 3622-3631.

Franks, S.J. (2011). Plasticity and evolution in drought avoidance and escape in the annual plant Brassica rapa. New Phytologist 190(1), 249-257.

Franks, S.J., Avise, J.C., Bradshaw, W.E., Conner, J.K., Etterson, J.R., Mazer, S.J., et al. (2008). The resurrection initiative: storing ancestral genotypes to capture evolution in action. BioScience 58(9), 870 873.

Franks, S.J., Sheina, S., and Weis, A.E. (2007). Rapid evolution of flowering time by an annual plant in response to a climate fluctuation. Proceedings of the National Academy of Sciences 104(4), 1278-1282.

Franks, S.J., Weber, J.J., and Aitken, S.N. (2014). Evolutionary and plastic responses to climate change in terrestrial plant populations. Evolutionary Applications 7(1), 123 -139. doi: doi: 10.1111/eva.12112.

Franks, S.J., and Weis, A.E. (2008). A change in climate causes rapid evolution of multiple life-history traits and their interactions in an annual plant. Journal of Evolutionary Biology 21, 1321-1334.

Garg, R., Tyagi, A., and Jain, M. (2012). Microarray analysis reveals overlapping and specific transcriptional responses to different plant hormones in rice Jain Plant Signaling \& Behavior 7(8).

Garrett, K., Thomas-Sharma, S., Forbes, G., and Nopsa, J. (2014). "Climate change and plant pathogen invasions," in Invasive Species and Global Climate Change, eds. L. Ziska \& J. Dukes. CABI).

Garrett, K.A., Dendy, S.P., Frank, E.E., Rouse, M.N., and Travers, S.E. (2006). Climate change effects on plant disease: genomes to ecosystems. Annual Review of Phytopathology 44, 489-509.

Gillis, J., Mistry, M., and Pavlidis, P. (2010). Gene function analysis in complex data sets using ErmineJ Nature Protocols 5(6), 1148-1159.

Glazebrook, J. (2005). Contrasting mechanisms of defense against biotrophic and necrotrophic pathogens. Annual Review of Phytopathology 43, 205 - 227.

Guo, Y., Samans, B., Chen, S., Kibret, K., Hatzig, S., Turner, N., et al. (2017). Drought-Tolerant Brassica rapa Shows Rapid Expression of Gene Networks for General Stress Responses and Programmed Cell Death 
Under Simulated Drought Stress. Plant Molecular Biology Reporter35, 416-430. doi: 10.1007/s11105017-1032-4.

Hartl, D.L., and Clark, A. (2007). Principles of Population Genetics.

Hoffmann, A., and Sgro, C. (2011). Climate change and evolutionary adaptation. Nature 470, 479 - 485.

Hoth, S., Morgante, M., Sanchez, J., Hanafey, M., Tingey, S., and Chua, N. (2002). Genome-wide gene expression profiling in Arabidopsis thaliana reveals new targets of abscisic acid and largely impaired gene regulation in the abi1-1 mutant. Journal of Cell Science 115(24), 4891 - 4900.

Huang, D., Wu, W., Abrams, S., and Cutler, A. (2008). The relationship of drought-related gene expression in Arabidopsis thaliana to hormonal and environmental factors. Journal of Experimental Botany 59(11), 2991 - 3007.

Jorgensen, T., and Emerson, B. (2008). Functional variation in disease resistance gene in populations of Arabidopsis thaliana. Molecular Ecology 17(22), 4912 - 4923.

Journot-Catalino, N., Somssich, I., Roby, D., and Kroj, T. (2006). The Transcription Factors WRKY11 and WRKY17 Act as Negative Regulators of Basal Resistance in Arabidopsis thaliana. The Plant Cell 18, 3289 $-3302$.

Karlsson, E., Baranowska, I., Wade, C., Hillbertz, N., Zody, M., Anderson, N., et al. (2007). Efficient mapping of mendelian traits in dogs through genome-wide association. Nature Genetics 39, 1321-1328.

Kofler, R., Orozco-terWengel, P., De Maio, N., and et al. (2011). PoPoolation: A toolbox for population genetic analysis of next generation sequencing data from pooled individuals. PLoS One 6, e15925.

Koike, S., Gladders, P., and Paulus, A. (2006). Vegetable Diseases: a Color Handbook. USA: Academic Press.

Laine, A., Burdon, J., Dodds, P., and Thrall, P. (2011). Spatial variation in disease resistance: from molecules to metapopulations. Journal of Ecology 99(1), 96-112.

Lamesch, P., Berardini, T., Li, D., Swarbreck, D., Wilks, C., Sasidharan, R., et al. (2011). The Arabidopsis Information Resource (TAIR): improved gene annotation and new tools. Nucleic Acids Research. doi: 10.1093/nar/gkr1090.

Lawrence, C., Mitchell, T., Craven, K., Cho, Y., Cramer, R., and Kim, K. (2008). At death's door: Alternaria pathogenecity mechanisms. Plant Pathology 24(2), 101-111.

$\mathrm{Li}, \mathrm{H}$., and Durbin, R. (2010). Fast and accurate long-read alignment with Burrows-Wheeler transform. Bioinformatcs 25, $589-595$. 
Li, H., Handsaker, B., Wysoker, A., Fennell, T., Ruan, J., Homer, N., et al. (2009). The sequence alignment/map format and SAMtools. Bioinformatcs 25, 2078 - 2079.

Liu, H., Yang, W., Dongcheng, L., Han, Y., Zhang, A., and Shaohua, L. (2011). Ectopic expression of a grapevine transcription factor $V v W R K Y 11$ contributes to osmotic stress tolerance in Arabidopsis. Molecular Biology Reports 38, 417 - 427.

Marra, J., Eo, S., Hale, M., Waser, P., and DeWoody, J. (2012). A priori and a posteriori approaches for finding genes of evolutionary interest in non-model species: Osmoregulatory genes in the kidney transcriptome of the desert rodent Dipodomys spectabilis (banner-tailed kangaroo rat). Comparative Biochemistry and Physiology Part D: Genomics and Proteomics 7(4), 328 - 339.

Mauch-Mani, B., and Mauch, F. (2005). The role of abscisic acid in plant-pathogen interactions. Current Opinion in Plant Biology 8, 409 - 414.

McDowell, J., and Dangl, J. (2000). Signal transduction in the plant immune response. Trends in Biochemical Sciences 25, 79 - 82.

McGrath, K., Dombrecht, B., Manners, J., PM, S., Edgar, C., Maclean, D., et al. (2005). Repressor- and activator-type ethylene response factors functioning in jasmonate signaling and disease resistance identified via a genome-wide screen of Arabidopsis transcription factor gene expression. Plant Physiology 139,949 - 959.

McKay, J., Rchards, J., and Mitchell-Olds, T. (2003). Genetics of drought adaptation in Arabidopsis thaliana: I. Pleiotropy contributes to genetic correlations among ecological traits. Molecular Ecology 12, 1137-1151.

Meena, P.D., Awasthi, R.P., Chattopadhyay, C., Kolte, S.J., and Kumar, A. (2010). Alternaria blight: a chronic disease in rapeseed-mustard. Journal of Oilseed Brassica 1(1), 1-11.

Mengiste, T. (2012). Plant immunity to necrotrophs. Annual Review of Phytopathology 50, 267-294.

Metcalf, C., and Mitchell-Olds, T. (2009). Life history in a model system: opening the black box with Arabidopsis thaliana. Ecology Letters 12, 593-600.

Mitchell-Olds, T., and Schmitt, J. (2006). Genetic mechanisms and evolutionary significance of natural variation in Arabidopsis. Nature 441, 947-952.

Mohr, P., and Cahill, D. (2007). Suppression by ABA of salicylic acid and lignin accumulation and the expression of multiple genes, in Arabidopsis infected with Pseudomonas syringae pv. tomato. Functional Integrative Genomics 7, 181 - 191.

Mole, S. (1994). Trade-offs and constraints in plant-herbivore defense theory: a life-history perspective. Oikos $71,3-12$. 
Mukherjee, A., Lev, S., Gepstein, S., and Horwitz, B. (2009). A compatible interaction of Alternaria brassicicola with Arabidopsis thaliana ecotype DiG: evidence for a specific transcriptional signature. BMC Plant Biology 9.

Nemhauser, J., Hong, F., and Chory, J. (2006). Different plant hormones regulate similar processes through largely nonoverlapping transcriptional responses. Cell 126, 467 - 475.

Nowicki, M., Nowakowska, M., Niezgoda, A., and Kozik, E. (2012). Alternaria black spot of crucifers: symptoms, importance of disease, and perspectives of resistance breeding. Vegetable Crops Research Bulletin 76, 5 - 19.

O'Hara, N., Rest, J., and Franks, S. (2016a). Factors affecting the disease severity of Alternaria blackspot in natural Brassica rapa populations on the California and Oregon coasts. Madrono 63, 249-257.

O'Hara, N., Rest, J., and Franks, S. (2016b). Increased susceptibility to fungal disease accompanies adaptation to drought in Brassica rapa. Evolution 70(1), 241-248.

Pandey, R., Kofler, R., Orozco-terWengel, P., Nolte, V., and Schlotterer, C. (2011). PoPoolation DB: a userfriendly web-based database for the retrieval of natural polymorphisms in Drosophila. Bmc Genetics 12(27).

Pautasso, M., Doring, T., Garbelotto, M., Lorenzo, P., and Jeger, M. (2012). Impacts of climate change on plant disease - opinions and trends. European Journal of Plant Pathology 133, 295 - 313.

Penuelas, J., and Filella, I. (2001). Responses to a warming world. Science 294, 793 - 795.

Qi, J., Yu, S., Zhang, F., Shen, X., Zhao, X., Yu, Y., et al. (2010). Reference Gene Selection for Real-Time Quantitative Polymerase Chain Reaction of mRNA Transcript Levels in Chinese Cabbage (Brassica rapa L. ssp. pekinensis). Plant Molecular Biology Reporter 28, 597-604.

R Core Team (2013). "R: A language and environment for statistical computing". (Vienna, Austria: R Foundation for Statistical Computing).

Rosas, U., Mei, Y., Xie, Q., Banta, J., Zhou, R., Seufferheld, G., et al. (2014). Variation in Arabidopsis flowering time associated with cis-regulatory variation in CONSTANS. Nature Communications 5. doi: $10.1038 /$ ncomms4651.

Rotem, J. (1994). The Genus Alternaria: Biology, Epidemiology, and Pathogenicity. St. Paul: APS Press.

Shinozaki, K., Yamaguchi-Shinozaki, K., and Seki, M. (2003). Regulatory network of gene expression in the drought and cold stress responses. Current Opinion in Plant Biology 6, 410-417.

Simms, E.L., and Rausher, M.D. (1987). Costs and benefits of plant resistance to herbivory. The American Naturalist 130(4), 570 - 581. 
Storey, J., and Tibshirani, R. (2003). Statistical significance for genomewide studies. Proceedings of the National Academy of Sciences 100, 9440.

Strimmer, K. (2008). fdrtool: a versatile R package for estimating local and tail area-based false discovery rates. Bioinformatcs 24, 1461 - 1462.

Taiz, L., and Zeiger, E. (2006). Plant Physiology. Sunderland, Mass.: Sinauer.

Tajima, F. (1989). Statistical method for testing the neutral mutation hypothesis by DNA polymorphism. Genetics 123, 585-595.

Thrall, P.H., and Burdon, J.J. (2003). Evolution of virulence in a plant host-pathogen metapopulation. Science 299, $1735-1737$.

Untergasser, A., Nijveen, H., Rao, X., Bisseling, T., Guerts, R., and Leunissen, J. (2007). Primer3Plus, an enhanced web interface to Primer3. Nucleic Acids Research 35(W71- 4).

van Wees, S., Chang, H., Zhu, T., and Glazebrook, J. (2003). Characterization of the early response of Arabidopsis to Alternaria brassicicola infection using expression profiling. Plant Physiology 132, 606 617.

Vance, C., Kirk, T., and Sherwood, R. (1980). Lignification as a mechanism of disease resistance. Annual Review of Phytopathology 18, 259 - 288.

Walsh, B. (2009). Quantitative genetics, version 3.0: where have we gone since 1987 and where are we headed? Genetica 136, 213-223.

Wang, X. (2011). The genome of the mesopolyploid crop species Brassica rapa. Nature Genetics.

Williams, G. (1957). Pleiotropy, natural selection, and the evolution of senescence. Evolution 11(4), 398 411.

Wu, K., Zhang, L., Zhou, C., Yu, C., and Chaikam, V. (2008). HDA6 is required for jasmonate response, senescence and flowering in Arabidopsis. Journal of Experimental Botany 59(2), 225-234.

Xie, D., Feys, B., James, S., Nieto-Rostro, M., and Turner, J. (1998). COI1: an Arabidopsis gene required for jasmonate-regulated defense and fertility. Science 280, $1091-1094$.

Zhou, C., Zhang, L., Duan, J., Miki, B., and Wu, K. (2005). HISTONE DEACETYLASE19 is involved in jasmonic acid and ethylene signaling of pathogen response in Arabidopsis. Plant cell 17, 1196-1204.

\section{Tables}

Table 1. The 20 most highly differentiated (high $\mathrm{F}_{\mathrm{ST}}$ ) necrotrophic fungal pathogen response genes. Significantly differentiated genes (based on q value) are bolded. Asterisks show significantly differentiated genes that are 
involved in both drought and pathogen stress response (van Wees et al., 2003; Mukherjee et al., 2009), as determined by expression analysis (1), or where genes have been shown to be COI1 dependent (2). Descriptions were retrieved from http://brassicadb.org/brad/. 


\begin{tabular}{|c|c|c|c|c|c|c|}
\hline $\begin{array}{l}\text { A. thaliana } \\
\text { ID }\end{array}$ & B. rapa ID & $\mathrm{F}_{\mathrm{ST}}$ & $\begin{array}{l}\mathrm{q} \\
\text { value }\end{array}$ & Gene Name & Notes & Description \\
\hline AT2G21520 & Bra030295 & 0.21 & $\begin{array}{l}1.75 \\
\times 10^{-} \\
5\end{array}$ & & 1 & $\begin{array}{l}\text { Sec14p-like } \\
\text { phosphatidylinositol transfer } \\
\text { family }\end{array}$ \\
\hline AT3G15210 & Bra038107* & 0.20 & $\begin{array}{l}7.30 \\
\times 10^{-} \\
5\end{array}$ & ERF4, RAP2.5 & 1,2 & $\begin{array}{l}\text { ethylene responsive element } \\
\text { binding factor } 4\end{array}$ \\
\hline AT5G54160 & Bra026320* & 0.20 & $\begin{array}{l}1.45 \\
\times 10- \\
4\end{array}$ & OMT1 & 1 & O-methyltransferase 1 \\
\hline AT2G02390 & Bra017417 & 0.19 & $\begin{array}{l}4.16 \\
\times 10^{-} \\
4\end{array}$ & GST18,GSTZ1 & 1 & $\begin{array}{l}\text { glutathione S-transferase } \\
\text { zeta } 1\end{array}$ \\
\hline AT4G01960 & Bra036302 & 0.17 & $\begin{array}{l}4.48 \\
\times 10^{-} \\
3\end{array}$ & & 1 & \\
\hline AT4G39270 & Bra033611* & 0.17 & $\begin{array}{l}5.63 \\
\times 10^{-} \\
3\end{array}$ & & 1 & $\begin{array}{l}\text { Leucine-rich repeat protein } \\
\text { kinase family }\end{array}$ \\
\hline AT4G31550 & Bra011282* & 0.16 & 0.02 & WRKY11 & 1 & $\begin{array}{l}\text { WRKY DNA-binding protein } \\
11\end{array}$ \\
\hline AT1G23040 & Bra012356* & 0.16 & 0.02 & & 1 & $\begin{array}{l}\text { hydroxyproline-rich } \\
\text { glycoprotein family protein }\end{array}$ \\
\hline AT2G33120 & Bra021828 & 0.16 & 0.02 & SAR1,VAMP722 & 1 & $\begin{array}{l}\text { synaptobrevin-related protein } \\
1\end{array}$ \\
\hline AT1G67980 & Bra033968 & 0.16 & 0.03 & CCOAMT & 1,2 & $\begin{array}{l}\text { caffeoyl-CoA 3-O- } \\
\text { methyltransferase }\end{array}$ \\
\hline AT2G17720 & Bra024495 & 0.15 & 0.05 & & 1 & $\begin{array}{l}\text { 2-oxoglutarate (2OG) and } \\
\text { Fe(II)-dependent oxygenase } \\
\text { superfamily protein }\end{array}$ \\
\hline AT1G45145 & Bra036335 & 0.15 & 0.06 & ATH5,LIV1,TRX5 & 1,2 & thioredoxin H-type 5 \\
\hline AT1G74020 & Bra003820 & 0.15 & 0.07 & SS2 & 1,2 & strictosidine synthase 2 \\
\hline AT1G23730 & Bra012384 & 0.15 & 0.07 & ВСАЗ & 1 & beta carbonic anhydrase 3 \\
\hline AT4G12720 & Bra012746 & 0.14 & 0.11 & GFG1,NUDT7 & 1 & MutT/nudix family protein \\
\hline AT2G02930 & Bra018543 & 0.14 & 0.14 & GST16,GSTF3 & 1 & glutathione S-transferase F3 \\
\hline
\end{tabular}




\begin{tabular}{|l|l|l|l|l|l|l|}
\hline AT1G10140 & Bra031702 & 0.14 & 0.16 & & 1,2 & $\begin{array}{l}\text { Uncharacterised conserved } \\
\text { protein UCP031279 }\end{array}$ \\
\hline AT3G02520 & Bra040592 & 0.14 & 0.17 & GF14 NU,GRF7 & 1 & general regulatory factor 7 \\
\hline AT5G65750 & Bra024417 & 0.14 & 0.19 & & 1 & $\begin{array}{l}\text { 2-oxoglutarate } \\
\text { dehydrogenase, E1 } \\
\text { component }\end{array}$ \\
\hline AT1G16520 & Bra026043 & 0.14 & 0.22 & & 1 & \\
\hline
\end{tabular}

\section{Figures}

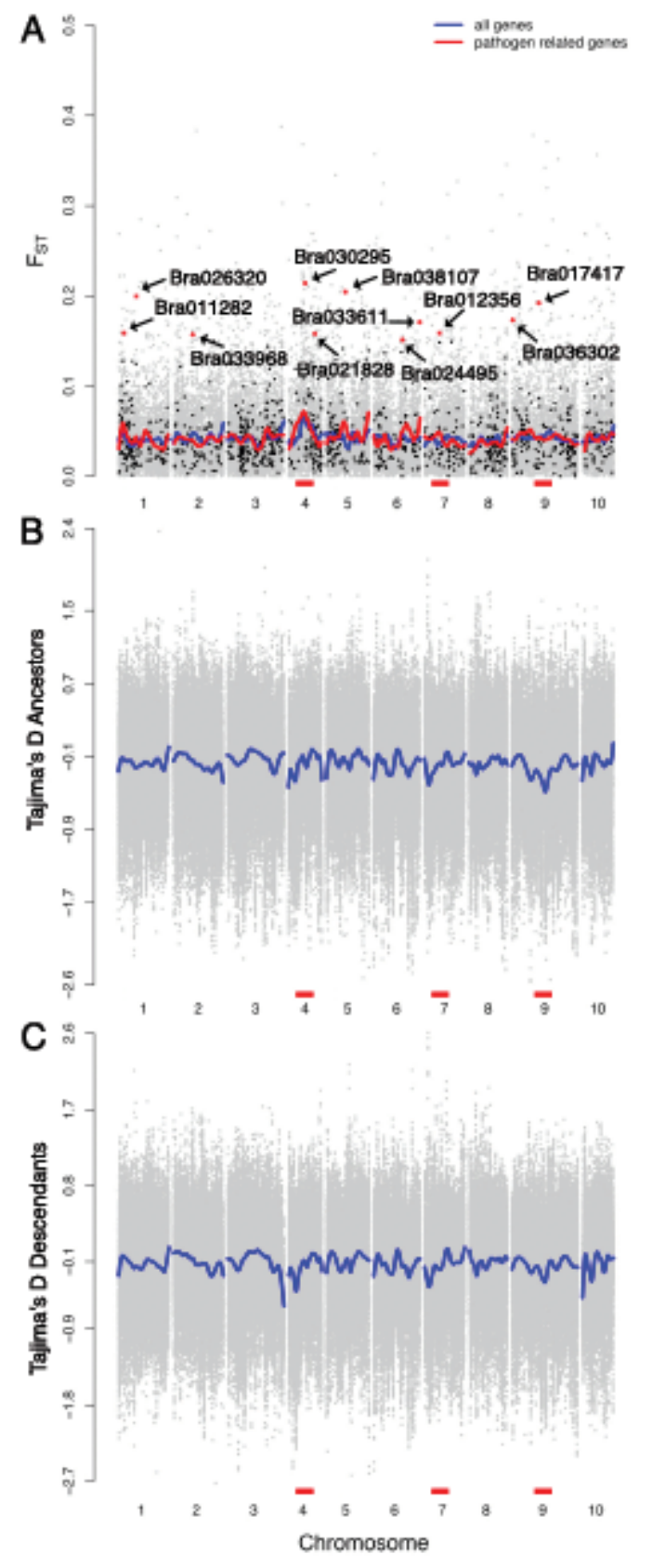


Differentiation at multiple loci across the genome in a Brassica rapa population after seven years of drought, as well as historical measures of selection. (A) FST, an estimate of allelic differentiation between the 1997 and 2004 populations, was calculated using 100kb sliding windows and then averaged for each gene (dots shown). Genes reported in the literature as involved in response to necrotrophic fungal infection (A. brassicicola) are shown in black. All other genes are shown in grey. For necrotrophic fungal pathogen response genes, significantly differentiated genes are labelled with red points and their gene ID. A LOESS trend line (span $=0.03$ ) is shown for all genes (blue) and just for necrotrophic fungal pathogen response genes (red). Tajima's $D$, a statistic estimating the effect of non-random processes from the site frequency spectrum, shows historical selection across the genome for the (B) ancestral population and (C) descendant populations. Analysis was conducted using a 100kb sliding window (dots shown are windows; LOESS trend line in blue). Red bars highlight regions containing necrotrophic pathogen related genes with a significant high FST that are also in an area with reduced Tajima's D (visual inspection). Regions with reduced Tajima's D may have been subject to a historical selective sweep.

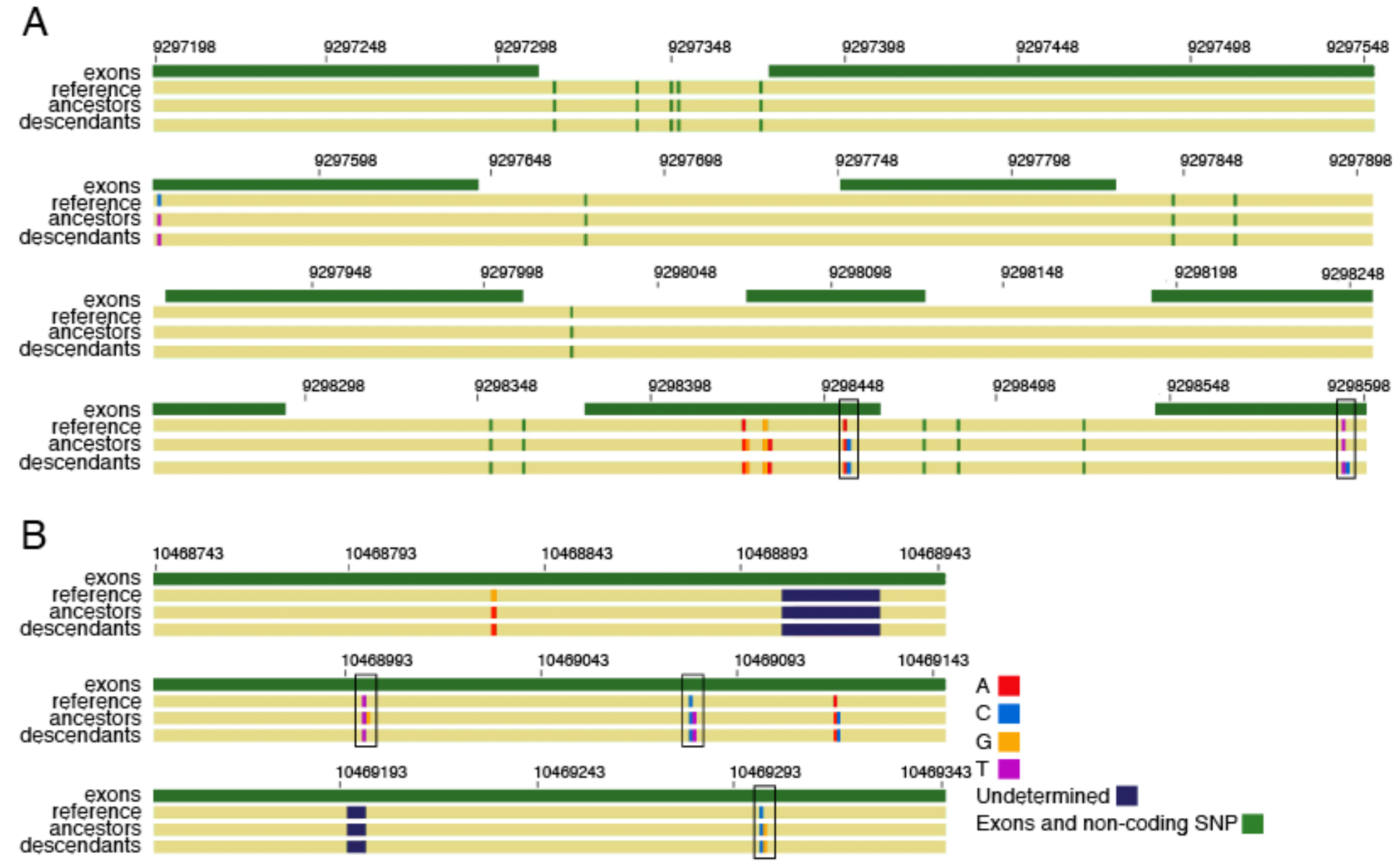

\section{Figure 2}

Evolution at non-synonymous sites in nectrophic fungal pathogen response genes in Brassica rapa after seven years of drought. Shown are alignments between B. rapa reference V. 1.18, ancestral B. rapa and descendant B. rapa for our 2 top candidate genes. Segregating sites are indicated by changes in the color 
of the bar across each alignment. (A) Bra030295 has 19 SNPs, 5 of which are in exons, and 2 of which are non-synonymous (black boxes). (B) Bra038107 had 5 segregating sites, all in the single exon, with 3 non-synonymous SNPs (black boxes).

\section{NFPR Genes $(1,182)$}

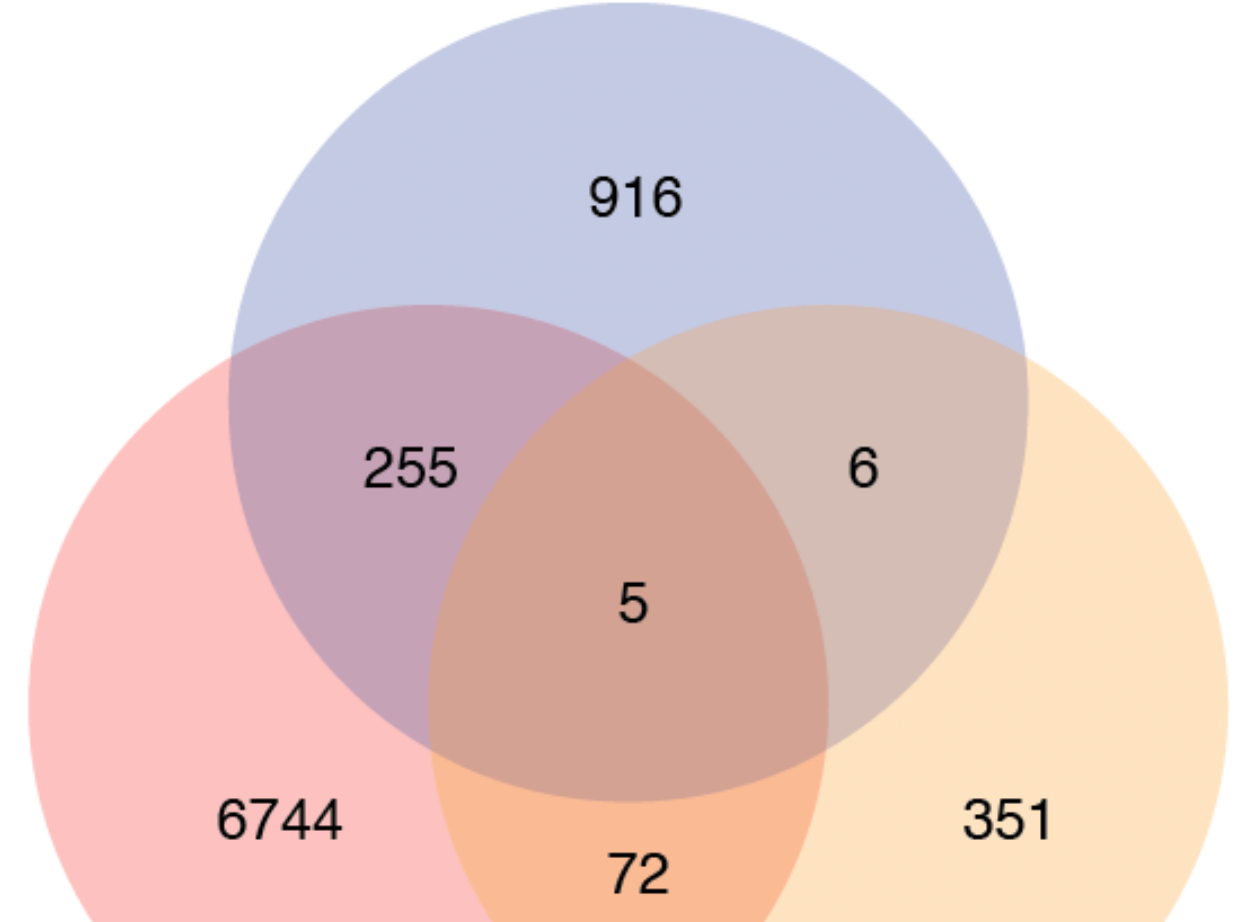

\section{Drought \\ Genes $(7,076)$}

\section{All High Fst Genes (434)}

\section{Figure 3}

Venn diagram showing gene databases used in this study for annotation including necrotrophic fungal pathogen response genes (NFPR), drought response genes (Drought), genes which evolved genome-wide (Sig Genes), and pleiotropic genes (overlap between NFPR and drought response) out of a total 35,202 genes.

\section{Supplementary Files}

This is a list of supplementary files associated with this preprint. Click to download.

- Additionalfile1.xIsx

- Additionalfile3.xlsx

- Additionalfile2.xlsx 